\section{SAT0192 MYOSITIS IN PATIENTS WITH PRIMARY SJÖGREN'S SYNDROME: DATA FROM A FRENCH NATIONWIDE SURVEY}

Camille Houssais ${ }^{1}$, Jean-Baptiste Noury ${ }^{2}$, Yves Allenbach ${ }^{3}$, Laure Gallay ${ }^{4}$ Florence Assan ${ }^{5}$, Olivier Benveniste ${ }^{3}$, Jonathan Broner ${ }^{6}$, Pierre Duffau ${ }^{7}$, Alexandra Espitia-Thibault ${ }^{8}$, Anne Grasland ${ }^{9}$, Sebastien Sanges ${ }^{10}$, Gilles Hayem ${ }^{11}$, Véronique Le Guern ${ }^{12}$, Nihal Martis ${ }^{13}$, Kuberaka Marianpillai ${ }^{14}$, Denis Mulleman ${ }^{15}$, Gaëtane Nocturne ${ }^{5}$, Alain Meyer ${ }^{16}$, Valerie DevauchellePensec ${ }^{1}$, Divi Cornec ${ }^{1}$, Dewi Guellec ${ }^{1} .{ }^{~}$ University Hospital, CERAINO, Brest, Rheumatology, Brest, France; ${ }^{2}$ University Hospital, CERAINO, Brest, Neurology, Brest, France; ${ }^{3}$ Pitié-Salpêtrière University Hospital, Paris, Internal Medicine and Clinical Immunology, Paris, France; ${ }^{4}$ Edouard Herriot University Hospital, Lyon, Lyon, France; ${ }^{5}$ Hôpitaux Universitaires Paris-Sud, Le kremlin-Bicêtre, France; ${ }^{6}$ Nîmes University Hospital, Internal Medicine, Nîmes, France; ${ }^{7}$ Centre Hospitalier Universitaire de Bordeaux, FHU ACRONIM, Bordeaux, France; ${ }^{8} \mathrm{Hôtel}-\mathrm{Dieu}, \mathrm{CHU}$ de Nantes, Internal Medicine, Nantes, France; ${ }^{9}$ Hôpital Louis Mourier, APHP, Colombes, France, Paris, France; ${ }^{10}$ Hopital Huriez, CHRU de Lille, Internal Medicine, Lille, France; ${ }^{11} \mathrm{CHU}$ Ambroise-Paré, AP-HP, Rheumatology, BoulogneBillancourt, France; ${ }^{12} \mathrm{CHU}$ Cochin, Paris, France; ${ }^{13} \mathrm{Côte}$ d'Azur University, Nice, France; ${ }^{14}$ Pitié-Salpêtrière University Hospital, Paris, Internal medicine and Clinical Immunology, Paris, France; ${ }^{15}$ University of Tours, Rheumatology, Tours, France; ${ }^{16} \mathrm{CHU}$ de Strasbourg, Département de Physiologie, Strasbourg, France

Background: Myositis is recognized as a manifestation of primary Sjögren's syndrome (pSS). Grading muscle involvement using the EULAR Sjögren Syndrome Disease Activity index (ESSDAl) does not require biopsy-proven active myositis or extensive immunological investigations. However, several studies have reported that a high proportion of pSSassociated myositis is either inclusion body myositis (IBM) or an overlap with idiopathic inflammatory myopathies.

Objectives: The objective of our study was to investigate the nature of inflammatory muscle involvement in a large sample of patients with pSS and concurrent myositis.

Methods: We conducted a French nation-wide survey among reference centers known for their experience in the field of systemic auto-immune diseases. Patients with physician-based diagnosis of pSS and inflammatory muscle involvement were included. They were categorized and subsequently described and compared according to the identification of an associated condition likely to explain inflammatory muscle involvement. Results: 60 cases of myositis in patients with pSS were identified. Patients were mostly female $(52 / 60)$ and pSS classification criteria were met in 43/60. Muscle biopsy was performed in 50/60 patients and showed inflammatory lesions in $80 \%$ of cases. An associated condition likely to explain myositis was identified in 31 patients: typical dermatomyositis rash $(n=8)$, MCTD-specific antibodies $(n=11)$, myositis specific antibodies $(n=10)$, and systemic lupus erythematosus, scleroderma or ANCA associated vasculitis specific antibodies in favor of an overlap syndrome $(n=7)$. Among the 29 patients without associated condition likely to explain myositis, 17 had a biopsy proven inflammatory muscle disease, showing histopathologic features consistent with IBM in 5 cases. 4 of the 5 patients with IBM histological features received corticosteroids and/or other immunosuppressive agents to treat muscle disease. Worsening of muscular involvement despite treatment was reported in these 4 patients. Comparative analysis showed a lower ESSDAI ( $p=0.001)$ among patients without identified associated condition likely to explain myositis. Conclusion: In most cases of concurrent pSS and myositis, inflammatory muscle involvement is explained by a context of overlap syndrome or has a histological pattern consistent with IBM. Extensive immunological investigations and muscle biopsy appear to be necessary in pSS patients with suspected inflammatory muscle involvement, in order to avoid misclassification and/or overtreatment.

Disclosure of Interests: Camille Houssais: None declared, Jean-baptiste Noury: None declared, Yves Allenbach: None declared, Laure Gallay: None declared, Florence Assan: None declared, Olivier Benveniste: None declared, Jonathan Broner: None declared, Pierre Duffau: None declared, Alexandra Espitia-Thibault: None declared, Anne Grasland: None declared, Sebastien SANGES: None declared, Gilles Hayem: None declared, Véronique LE GUERN: None declared, Nihal Martis: None declared, Kuberaka Marianpillai: None declared, Denis Mulleman Speakers bureau: Pfizer, Novartis, Grifols, Gaëtane Nocturne: None declared, alain meyer: None declared, Valerie Devauchelle-Pensec Grant/research support from: RocheChugai, Speakers bureau: MSD, BMS, UCB, Roche, Divi Cornec: None declared, Dewi Guellec: None declared DOI: 10.1136/annrheumdis-2019-eular.3474

\section{SAT0193 \\ THE USE OF A SYMPTOM CHECKLIST TO RECOGNISE PATIENTS WITH COMORBID FIBROMYALGIA ON A BACKGROUND OF SYSTEMIC LUPUS ERYTHEMATOSUS}

Frank Huang ${ }^{1},{ }^{1,2}$ Sean O'neill ${ }^{3}$, Ray Fang ${ }^{1}$, Matthew Nguyen ${ }^{1},{ }^{1,2}$ Kathryn Gibson $^{3}$. ${ }^{1}$ University of New South Wales, Sydney, Australia; ${ }^{2}$ Liverpool Hospital, Sydney, Australia; ${ }^{3}$ Ingham Research Institute, Sydney, Australia

Background: Fibromyalgia (FM) and systemic lupus erythematosus (SLE) are both characterised by non-specific symptoms such as pain and fatigue. To make diagnosis even more challenging, fibromyalgia is overrepresented in the SLE population.

Objectives: To identify which symptoms can discriminate between patients with comorbid FM and patients without comorbid FM in the SLE population, using a routinely distributed questionnaire.

Methods: Patients with SLE $(n=88)$ completed a Multi-Dimensional Health Assessment Questionnaire (MDHAQ) [1] and the 2011 FM Criteria questionnaire [2]. FM status was determined using the 2016 modification of the 2010/2011 FM criteria [3]. The MDHAQ contains a 60-item symptom checklist section, giving a score of 0-60. Patients with complete data were analysed for specific symptoms that were discriminating for FM using student's $t$ test with Bonferroni correction.

Results: SLE patients with FM reported a higher prevalence of positive responses for every symptom except for 'dark or bloody stools' and 'burning in sexual organs'. Nineteen symptoms demonstrated a significant difference between those with and without FM. The most discriminating symptoms were muscle pain, swelling (of hands, ankles and in other joints), back pain, neck pain, problems with thinking and dry mouth (all $p<0.0001$ ). Grouping the above 8 symptoms, a cut-off of $>4$ gave a sensitivity of $91 \%$ and specificity of $88 \%$, correctly classifying $89 \%$ of patients when compared to FM criteria. Overall, patients with FM reported a mean total of 23.6 items on the symptom checklist, compared to 8.09 for patients without FM.

Conclusion: The symptom checklist section of the MDHAQ can provide clues that help discriminate between SLE patients with and without FM Clinicians can find valuable information in the specific symptoms experienced by the patient, as well as the overall number of symptoms. Grouping the discriminatory symptoms together in the questionnaire may assist clinicians to consider the diagnosis of FM more easily in patients with SLE.

\section{REFERENCES}

[1] Pincus, T., Y. Yazici, and M. Bergman, Development of a multi-dimensional health assessment questionnaire (MDHAQ) for the infrastructure of standard clinical care. Clinical \& Experimental Rheumatology, 2005. 23(5 Suppl 39): p. S19-28.

[2] Wolfe, F., et al., Fibromyalgia criteria and severity scales for clinical and epidemiological studies: a modification of the ACR Preliminary Diagnostic Criteria for Fibromyalgia. J Rheumatol, 2011. 38(6): p. 1113-22.

[3] Wolfe, F., et al. 2016 Revisions to the 2010/2011 fibromyalgia diagnostic criteria. in Seminars in arthritis and rheumatism. 2016. Elsevier.

Disclosure of Interests: Frank Huang: None declared, Sean O'Neill: None declared, Ray Fang: None declared, Matthew Nguyen: None declared, Kathryn Gibson Grant/research support from: UCB, Abbvie, Speakers bureau: UCB, Janssen

DOI: 10.1136/annrheumdis-2019-eular.6665

\section{SAT0194 EVALUATION OF PSYCHOMETRIC PROPERTIES OF THE PATIENT-REPORTED OUTCOMES MEASUREMENT INFORMATION SYSTEM (PROMIS) PHYSICAL FUNCTION 10-ITEM SHORT FORM IN SYSTEMIC LUPUS ERYTHEMATOSUS}

Zara Izadi, Julia Kay, Gabriela Schmajuk, Jinoos Yazdany. University of California, San Francisco, San Francisco, United States of America

Background: While the PROMIS (Patient-Reported Outcomes Measurement Information System) physical function short form 10a (PF10a) is both practical and acceptable for implementation in routine clinical practice, its psychometric properties have not been evaluated in Systemic Lupus Erythematosus (SLE).

Objectives: The objectives of our study were to examine the validity and responsiveness of PF10a in SLE among a racially/ethnically diverse clinic population and develop estimates of the minimally important difference (MID). 